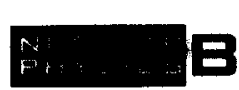

Nuclear Physics B 550 (1999) 151-168

www.elsevier.nl/locate/npe

\title{
Non-commutative open string and D-brane
}

\author{
Chong-Sun $\mathrm{Chu}^{\mathrm{a}, 1}$, Pei-Ming $\mathrm{Ho}^{\mathrm{b}, 2}$ \\ ${ }^{a}$ Institute of Physics, University of Neuchâtel, CH-2000 Neuchâtel, Switzerland \\ ${ }^{b}$ Department of Physics, National Taiwan University, Taipei 10764, Taiwan, R.O.C. \\ Received 15 January 1999; revised 10 March 1999; accepted 18 March 1999
}

\begin{abstract}
In this paper we consider the quantization of open strings ending on D-branes with a background $B$ field. We find that space-time coordinates of the open string end-points become noncommutative, and correspondingly the D-brane world-volume also becomes non-commutative. This provides a string theory derivation and generalization of the non-commutativity obtained previously in the $\mathrm{M}$ (atrix) model compactification. For $\mathrm{D} p$-branes with $p \geqslant 2$ our results are new and agree with that of $\mathrm{M}$ (atrix) theory for the case of $A=0$ (where $A$ is the world-volume gauge field) if the $T$-duality radii are used. (C) 1999 Elsevier Science B.V. All rights reserved.
\end{abstract}

PACS: 11.25.Db; 11.30.Ly; 11.15.Tk

Keywords: D-brane; String theory in backgrounds; Non-commutative geometry

\section{Introduction}

$\mathrm{M}$ (atrix) theory [1] compactified on a torus is described by a supersymmetric YangMills (SYM) theory living on the dual torus $[1,2]$. However, this simple picture no longer holds when a background field is present. It was shown by Connes, Douglas and Schwarz in [3] that for the $\mathrm{M}$ (atrix) model compactified on a $T^{2}$, non-commutative SYM arises naturally if there is a background field $C_{-12}$. This is justified from the Dstring point of view [4] by dualizing one cycle of the torus. Later it was demonstrated directly how non-commutativity arises from the D0-brane point of view $[5,6]$.

It was then also suggested in [7] by Hofman and Verlinde that a D-brane worldvolume is non-commutative already in string theory before taking the $\mathrm{M}$ (atrix) model

\footnotetext{
I E-mail: cschu@sissa.it

${ }^{2}$ E-mail: pmho@phys.ntu.edu.tw 
limit. Taking a similar approach as in [8], we demonstrate in this paper that the noncommutativity on D-brane world-volume has an explanation in terms of open string quantization in background fields.

We find that the end-points of the string have non-commutative coordinates and therefore we infer that the D-brane world-volume is a non-commutative space. The noncommutativity derived from string quantization is shown to agree with the results of M(atrix) model compactified on torus when the $U(1)$ field strength $F$ on the D-brane world-volume vanishes. In general the non-commutativity is determined by the gaugeinvariant combination $\mathcal{F}=(B-F)$, instead of just the NS two-form $B$ field. We show that the D-brane world-volume does not need to be a compact space in order to have non-commutativity.

We also give explicit formula telling how the string theory data (the gauge-invariant background field strength $\mathcal{F}$ ) should be encoded in the non-commutative world-volume of a $D p$-brane for $p \geqslant 2$, generalizing previous results.

\section{Classical action}

Consider a fundamental string ending on a $\mathrm{D} p$-brane, the bosonic part of the action is $[9,10]$

$$
\begin{aligned}
S_{B}= & \frac{1}{4 \pi \alpha^{\prime}} \int_{\Sigma} d^{2} \sigma\left[g^{\alpha \beta} G_{\mu \nu} \partial_{\alpha} X^{\mu} \partial_{\beta} X^{\nu}+\epsilon^{\alpha \beta} B_{\mu \nu} \partial_{\alpha} X^{\mu} \partial_{\beta} X^{\nu}\right] \\
& +\frac{1}{2 \pi \alpha^{\prime}} \oint_{\partial \Sigma} d \tau A_{i}(X) \partial_{\tau} X^{i}
\end{aligned}
$$

where $A_{i}, i=0,1, \ldots, p$, is the $U(1)$ gauge field living on the D $p$-brane. Here the string background is

$$
G_{\mu \nu}=\eta_{\mu \nu}, \quad \Phi=\text { constant }, \quad H=d B=0 .
$$

Variation of the action yields the equations of motion for a free field,

$$
\left(\partial_{\tau}^{2}-\partial_{\sigma}^{2}\right) X^{\mu}=0
$$

and the following boundary conditions at $\sigma=0, \pi$ :

$$
\begin{aligned}
\partial_{\sigma} X^{i}+\partial_{\tau} X^{j} \mathcal{F}_{j}{ }^{i} & =0, \quad i, j=0,1, \ldots, p, \\
X^{a} & =x_{0}^{a}, \quad a=p+1, \ldots, 9 .
\end{aligned}
$$

Here

$$
\mathcal{F}=B-d A=B-F
$$

is the modified Born-Infeld field strength and $x_{0}^{a}$ is the location of the D-brane. Indices are raised and lowered by $\eta_{i j}=(-,+, \ldots,+)$. 
If both ends of a string are attached to the same $\mathrm{D} p$-brane, the last term in (1) can be written as

$$
\frac{-1}{4 \pi \alpha^{\prime}} \int_{\Sigma} d^{2} \sigma \epsilon^{\alpha \beta} F_{i j} \partial_{\alpha} X^{i} \partial_{\beta} X^{j}
$$

Furthermore consider the case $B=\sum_{i, j=0}^{p} B_{i j} d X^{i} d X^{j}$, then the action (1) can be written as

$$
S_{B}=-\int d \tau L=\frac{1}{4 \pi \alpha^{\prime}} \int d^{2} \sigma\left[g^{\alpha \beta} \eta_{\mu \nu} \partial_{\alpha} X^{\mu} \partial_{\beta} X^{\nu}+\epsilon^{\alpha \beta} \mathcal{F}_{i j} \partial_{\alpha} X^{i} \partial_{\beta} X^{j}\right]
$$

Note that for open strings $B$ and $F$ always appear together in the combination $\mathcal{F}=B-F$, which is invariant under both gauge transformations for the one-form gauge field $A$,

$$
A \rightarrow A+d A, \quad B \rightarrow B
$$

and for the two-form gauge field $B$,

$$
B \rightarrow B+d A, \quad A \rightarrow A+\Lambda .
$$

We will be interested in the consequence of (4) due to the presence of a constant background field $\mathcal{F}$ along the directions of the world-volume of the D-brane. A remark about the boundary conditions is in order. In the usual boundary state formalism, a Dbrane is described by the boundary state $[11,12]$. The boundary conditions for the open string ending on the D-brane are translated into conditions (the overlapping conditions) satisfied by the boundary state. In particular, the boundary conditions (4) and (5) are not implemented as operator constraints, but rather implemented as constraints on the space of boundary states.

In this paper, we are interested in seeing the origin of the non-commutativity on the D-brane world-volume field theory due to the presence of a non-trivial two-form background field $\mathcal{F}$. Instead of using the boundary state formalism, we will use a more direct operator approach by implementing the conditions (4) and (5) as operator constraints and investigate their consequences. This approach is intuitively clearer and easier to make connection with previous results $[3,4,13,14]$ on non-commutative gauge theory as most results in the literature are expressed in the operator language. Modulo technical details, we expect that the two approaches give completely equivalent results because of the standard duality between operators and states. It would be very interesting to see explicitly how non-commutativity arises in the boundary state formalism.

The general solution of $X^{k}$ to the equations of motion is

$$
X^{k}=x_{0}^{k}+\left(a_{0}^{k} \tau+b_{0}^{k} \sigma\right)+\sum_{n \neq 0} \frac{e^{-i n \tau}}{n}\left(i a_{n}^{k} \cos n \sigma+b_{n}^{k} \sin n \sigma\right) .
$$

Substituting (11) into the boundary conditions (4), we get

$$
b_{n}^{k}+a_{n}^{j} \mathcal{F}_{j}^{k}=0, \quad \text { for all } n
$$


Eliminating $b_{n}^{k}$ and denoting $a_{0}^{k}=p_{0}^{k}$, we get

$$
X^{k}=x_{0}^{k}+\left(p_{0}^{k} \tau-p_{0}^{j} \mathcal{F}_{j}^{k} \sigma\right)+\sum_{n \neq 0} \frac{e^{-i n \tau}}{n}\left(i a_{n}^{k} \cos n \sigma-a_{n}^{j} \mathcal{F}_{j}{ }^{k} \sin n \sigma\right) .
$$

Similarly we can solve the equations of motion and boundary conditions for $X^{a}$ and get

$$
X^{a}=x_{0}^{a}+b^{a} \sigma+\sum_{n \neq 0} \frac{e^{-i n \tau}}{n} a_{n}^{a} \sin n \sigma, \quad a=p+1, \ldots, 9,
$$

where $x_{0}^{a}+\pi b^{a}$ is the location of the D-brane to which the other end-point of the open string is attached. If both ends of the string end on the same brane then $b^{a}=0$.

The canonical momentum from the action (1) is given by

$$
\begin{aligned}
& 2 \pi \alpha^{\prime} P^{k}(\tau, \sigma)=\partial_{\tau} X^{k}+\partial_{\sigma} X^{j} \mathcal{F}_{j}{ }^{k}, \\
& 2 \pi \alpha^{\prime} P^{a}(\tau, \sigma)=\partial_{\tau} X^{a} .
\end{aligned}
$$

Substituting (13) into the above expressions, we get

$$
\begin{aligned}
& 2 \pi \alpha^{\prime} P^{k}(\tau, \sigma)=\left\{p_{0}^{l}+\sum_{n \neq 0} a_{n}^{l} e^{-i n \tau} \cos n \sigma\right\} M_{l}^{k}, \\
& 2 \pi \alpha^{\prime} P^{a}(\tau, \sigma)=-i \sum_{n \neq 0} e^{-i n \tau} a_{n}^{a} \sin n \sigma,
\end{aligned}
$$

where

$$
M_{i j}=\eta_{i j}-\mathcal{F}_{i}^{k} \mathcal{F}_{k j}
$$

One can check that the total momenta

$$
P_{\mathrm{tot}}^{k}(\tau)=\int_{0}^{\pi} d \sigma P^{k}(\tau, \sigma)=\frac{1}{2 \alpha^{\prime}} p_{0}^{l} M_{l}^{k}
$$

are constants of motion.

The center of mass coordinates of the string is

$$
x_{\mathrm{cm}}^{k}(\tau)=\frac{1}{\pi} \int_{0}^{\pi} d \sigma X^{k}(\tau, \sigma)
$$

It is

$$
x_{\mathrm{cm}}^{k}=x_{0}^{k}+p_{0}^{k} \tau-\frac{\pi}{2} p_{0}^{j} \mathcal{F}_{j}^{k}-\sum_{n \neq 0} \frac{e^{-i n \tau}}{n^{2}}\left(1-(-1)^{n}\right) a_{n}^{j} \mathcal{F}_{j}{ }^{k} .
$$

The Hamiltonian is defined by

$$
H_{B}=\int d \sigma P_{\mu} X^{\mu}-L
$$


and is found to be

$$
H_{B}=\frac{1}{4 \pi \alpha^{\prime}} \int d \sigma\left(\left(\partial_{\tau} X\right)^{2}+\left(\partial_{\sigma} X\right)^{2}\right) .
$$

Using the mode expansion (13), (14), we find

$$
H_{B}=\frac{1}{4 \alpha^{\prime}}\left(M_{i j} p_{0}^{i} p_{0}^{j}+b^{a} b^{a}+\sum_{n \neq 0}\left(M_{i j} a_{n}^{i} a_{-n}^{j}+a_{n}^{a} a_{-n}^{a}\right)\right) \text {. }
$$

\section{Quantization}

The quantization of $X(\tau, \sigma)$ has to be different from the usual canonical commutation relations for free fields because the standard equal time commutation relations are inconsistent with the boundary conditions (4) [15,8]. To see this, we use (4) and (15) and obtain

$$
2 \pi \alpha^{\prime} P^{k}(\tau, 0) \mathcal{F}_{k}{ }^{i}=-\partial_{\sigma} X^{j}(\tau, 0) M_{j}{ }^{i} .
$$

It follows that

$$
2 \pi \alpha^{\prime}\left[P^{k}(\tau, 0), P^{j}(\tau, \sigma)\right] \mathcal{F}_{k}^{i}=-\left[\partial_{\sigma} X^{k}(\tau, 0), P^{j}(\tau, \sigma)\right] M_{k}^{i} .
$$

This relation makes it impossible to impose both the following standard canonical commutation relations consistently:

$$
\begin{aligned}
& {\left[X^{i}(\tau, \sigma), P_{j}\left(\tau, \sigma^{\prime}\right)\right]=i \delta_{j}^{i} \delta\left(\sigma-\sigma^{\prime}\right),} \\
& {\left[P_{i}(\tau, \sigma), P_{j}\left(\tau, \sigma^{\prime}\right)\right]=0}
\end{aligned}
$$

A similar situation also occurred in the usual quantization of Maxwell field in the Coulomb gauge. There one finds that the gauge-fixing condition is not consistent with the standard canonical quantization and one has to modify the quantization in a consistent manner. We will do the same in the following. We expect that our quantization procedure to be equivalent to the constraint quantization of Dirac. ${ }^{3}$ The conflict between the usual relations for free fields in this case was first realized in $[15,8]$. However, their quantization does not agree with ours. (See in particular Eq. (60) below and the discussions before it.)

Let us first review the usual quantization procedure and then we will see that there is a natural generalization of the usual canonical quantization procedure that takes care of our case. The usual way to quantize a classical system is to start with the symplectic structure on the phase space. For example, in the standard string case with $B=A=0$, one first rewrites the action in the Hamiltonian form

$$
-\int d^{2} \sigma\left(P_{\mu} \dot{X}^{\mu}-\mathcal{H}\right)
$$

\footnotetext{
${ }^{3}$ We are grateful to $M$. Cederwall for informing us that this is indeed the case.
} 
where $\mathcal{H}$ is the Hamiltonian density. The symplectic form

$$
\Omega \text { (fields) }=\int d \sigma \mathbf{d} P_{\mu} \mathbf{d} X^{\mu}
$$

is extracted from the first piece [16] and it defines the standard Poisson bracket for the fields $X^{\mu}, P^{\mu}$

$$
\begin{aligned}
& {\left[X^{\mu}(\tau, \sigma), P_{\nu}\left(\tau, \sigma^{\prime}\right)\right]_{\mathrm{PB}}=\delta_{\nu}^{\mu} \delta\left(\sigma-\sigma^{\prime}\right),} \\
& {\left[P_{\mu}(\tau, \sigma), P_{\nu}\left(\tau, \sigma^{\prime}\right)\right]_{\mathrm{PB}}=0, \quad\left[X_{\mu}(\tau, \sigma), X_{\nu}\left(\tau, \sigma^{\prime}\right)\right]_{\mathrm{PB}}=0,}
\end{aligned}
$$

which are the standard equal time commutation relations for free fields. Plugging in the mode expansions (13), (14), (17) and (18) of $X^{\mu}$ and $P^{\mu}$ for the standard case of $B=A=0$, one gets the usual commutation relations for the modes.

One can also derive the Poisson structure for the modes directly without going through the fields. To do this, one just have to follow the above steps by first plugging in the mode expansions for $X^{\mu}$ and $P^{\mu}$ and then evaluate (31). The result is

$$
\Omega(\text { modes })=\frac{1}{2 \alpha^{\prime}}\left\{\eta_{i j} \mathbf{d} p_{0}^{i} \mathbf{d} x_{0}^{j}+\sum_{n>0} \frac{-i}{n}\left(\eta_{i j} \mathbf{d} a_{n}^{i} \mathbf{d} a_{-n}^{j}+\mathbf{d} a_{n}^{a} \mathbf{d} a_{-n}^{a}\right)\right\} .
$$

This implies the same standard commutation relations for the modes and the two approaches are completely equivalent.

All of these are quite standard. In our case, because of the boundary conditions (4), we saw that it is inconsistent to impose (32), (33), even in the Poisson limit. We propose to use

$$
\tilde{\Omega}(\text { modes })=\left\langle\int d \sigma \mathbf{d} P_{\mu} \mathbf{d} X^{\mu}\right\rangle,
$$

where $\langle\cdot\rangle$ is the time average

$$
\langle O\rangle=\lim _{T \rightarrow \infty} \frac{1}{2 T} \int_{-T}^{T} O d \tau
$$

as the definition of the symplectic structure for the modes in general. A remark on this definition is in order. Usually one takes the symplectic form to be given by $\int d \sigma \mathbf{d} P_{\mu} \mathbf{d} X^{\mu}$ because the mode expansion of $X$ is given by an orthogonal basis of spatial functions. However, generically the modes are orthogonal only as functions on the whole spacetime, but not necessarily orthogonal in spatial dependence. In our case, due to the boundary conditions, the spatial dependence of the mode expansion is not orthogonal, so if we do not average over time we will be left with $\tau$-dependence in the symplectic form for the modes. This is not consistent. Here we talk about string as an example, but in general one can use (35) for a generic system. It is easy to see that (35) reproduces the usual results for all typical cases in quantum field theory. Applying to our particular case with the string defined by (1)-(5), eq. (35) defines a consistent quantization. From 
another viewpoint, the definition (35) is very natural because the time integration is already there in the action from which the symplectic form is extracted. We will give further justifications of our results later by showing that in our quantization, the correct time evolution (48) of $X$ and the standard Virasoro algebra is obtained.

Now substituting (13) and (15), we find

$$
\tilde{\Omega}=\frac{1}{2 \alpha^{\prime}}\left\{M_{i j} \mathbf{d} p_{0}^{i}\left(\mathbf{d} x_{0}^{j}+\frac{\pi}{2} \mathcal{F}^{j}{ }_{k} \mathbf{d} p_{0}^{k}\right)+\sum_{n>0} \frac{-i}{n}\left(M_{i j} \mathbf{d} a_{n}^{i} \mathbf{d} a_{-n}^{i}+\mathbf{d} a_{n}^{a} \mathbf{d} a_{-n}^{a}\right)\right\} .
$$

As a consistency check, it is easy to see that this reduces to (34) in the usual situation of $\mathcal{F}=0$. This expression can be simplified if we shift the range of $\sigma$ by $\pi / 2$ so that $\sigma \in[-\pi / 2, \pi / 2]$ and define the coordinates for the center of a string

$$
\bar{x}_{0}^{i}=x_{0}^{i}+\frac{\pi}{2} \mathcal{F}_{i j} p_{0}^{j} \text {. }
$$

It implies the following commutation relations:

$$
\begin{aligned}
& {\left[\bar{x}_{0}^{i}, \bar{x}_{0}^{j}\right]=\left[p_{0}^{i}, p_{0}^{j}\right]=0,} \\
& {\left[\bar{x}_{0}^{i}, p_{0}^{j}\right]=i 2 \alpha^{\prime} M^{-1 i j},} \\
& {\left[a_{n}^{i}, \bar{x}_{0}^{j}\right]=\left[a_{n}^{i}, p_{0}^{j}\right]=0,} \\
& {\left[a_{m}^{i}, a_{n}^{j}\right]=2 \alpha^{\prime} m M^{-1 i j} \delta_{m+n},} \\
& {\left[a_{m}^{a}, a_{n}^{b}\right]=2 \alpha^{\prime} m \delta^{a b} \delta_{m+n} .}
\end{aligned}
$$

In terms of $x_{0}^{i}$ it is

$$
\begin{aligned}
& {\left[x_{0}^{i}, p_{0}^{j}\right]=i 2 \alpha^{\prime} M^{-1 i j},} \\
& {\left[x_{0}^{i}, x_{0}^{j}\right]=i 2 \pi \alpha^{\prime}\left(M^{-1} \mathcal{F}\right)^{i j},} \\
& {\left[p_{0}^{i}, p_{0}^{j}\right]=0 .}
\end{aligned}
$$

Reality of the fields $X^{\mu}$ implies that $x_{0}^{\mu}, b^{a}, p_{0}^{k}$ are real and

$$
a_{n}^{\mu \dagger}=a_{-n}^{\mu}
$$

As a consistency check, it is easy to see that the above commutation relations respect this reality structure.

Using (25) we can check that

$$
\left[H, X^{\mu}(\tau, \sigma)\right]=-i \partial_{\tau} X^{\mu}(\tau, \sigma), \quad\left[H, P^{\mu}(\tau, \sigma)\right]=-i \partial_{\tau} P^{\mu}(\tau, \sigma),
$$

and that the center of mass coordinate $x_{\mathrm{cm}}^{k}$ is conjugate to the total momentum $P_{\mathrm{tot}}^{k}$ in the usual sense,

$$
\begin{aligned}
& {\left[x_{\mathrm{cm}}^{j}, P_{\mathrm{tot}}^{k}\right]=i \eta^{j k},} \\
& {\left[x_{\mathrm{cm}}^{j}, x_{\mathrm{cm}}^{k}\right]=0, \quad\left[P_{\mathrm{tot}}^{j}, P_{\mathrm{tot}}^{k}\right]=0 .}
\end{aligned}
$$

The space-time coordinate $X(\tau, \sigma)$ and momentum $P(\tau, \sigma)$ are derived concept and their equal time commutators can be derived. It is 


$$
\begin{aligned}
& {\left[P^{i}(\tau, \sigma), P^{j}\left(\tau, \sigma^{\prime}\right)\right]=0} \\
& {\left[X^{k}(\tau, \sigma), X^{l}\left(\tau, \sigma^{\prime}\right)\right]=-2 i \alpha^{\prime}\left(M^{-1} \mathcal{F}\right)^{k l}\left[\left(\sigma+\sigma^{\prime}-\pi\right)+\sum_{n \neq 0} \frac{1}{n} \sin n\left(\sigma+\sigma^{\prime}\right)\right]} \\
& {\left[X^{i}(\tau, \sigma), P^{j}\left(\tau, \sigma^{\prime}\right)\right]=i \eta^{i j} \cdot \frac{1}{\pi}\left(1+\sum_{n \neq 0} \cos n \sigma \cos n \sigma^{\prime}\right)}
\end{aligned}
$$

Letting $\theta=\left(\sigma+\sigma^{\prime}\right)$, the infinite series on the right-hand side of (52) is the Fourier expansion

$$
\sum_{n \neq 0} \frac{1}{n} \sin n \theta=\theta-\pi
$$

for $\theta \in(0,2 \pi)$. But at the boundary we have

$$
\sum_{n \neq 0} \frac{1}{n} \sin n \theta=0
$$

for $\theta=0,2 \pi$. Therefore,

$$
\left[X^{k}(\tau, \sigma), X^{l}\left(\tau, \sigma^{\prime}\right)\right]=0
$$

for all values of $\sigma$ and $\sigma^{\prime}$ except that when $\sigma=\sigma^{\prime}=0$, it is

$$
\left[X^{k}(\tau, 0), X^{l}(\tau, 0)\right]=2 \pi i \alpha^{\prime}\left(M^{-1} \mathcal{F}\right)^{k l},
$$

and that when $\sigma=\sigma^{\prime}=\pi$, it is

$$
\left[X^{k}(\tau, \pi), X^{l}(\tau, \pi)\right]=-2 \pi i \alpha^{\prime}\left(M^{-1} \mathcal{F}\right)^{k l} .
$$

The infinite series on the right-hand side of (53)

$$
\frac{1}{\pi}\left(1+\sum_{n \neq 0} \cos n \sigma \cos n \sigma^{\prime}\right)
$$

is the Fourier expansion of the delta function $\delta\left(\sigma-\sigma^{\prime}\right)$ for functions defined on the interval $[0, \pi]$ with vanishing derivatives on the boundary.

Thus we see that the commutation relations are the standard ones for any point in the interior of the open string. At the two end points of the open string where the D-branes sit, we find the space-time coordinates to be non-commutative.

The non-commutativity of the space-time coordinates of an open string was first suggested in Ref. [8]. However, the commutation relation they found was

$$
\left[X^{i}(\tau, \sigma), X^{j}\left(\tau, \sigma^{\prime}\right)\right]=2 \pi i \mathcal{F}^{i j} \theta\left(\sigma-\sigma^{\prime}\right) .
$$

This expression is not well defined for $\sigma=\sigma^{\prime}$ and it does not agree with our result.

Note that $\left(M^{-1} \mathcal{F}\right)^{k l}$ is anti-symmetric as required. These are different from the usual commutation relations for free fields. Since the end-point of the string can be identified 
with the D-brane world-volume, we will use this relation in the next section for the points $\sigma=0, \pi$ to make statements about the non-commutativity on the D-brane world-volume and to compare them with what we know from $M$ (atrix) model compactification.

In general, one can define for a $\mathrm{D} p$-brane the following number operator:

$$
N_{n}=N_{n}^{\prime}+N_{n}^{\prime \prime}, \quad n \neq 0,
$$

where $N_{n}^{\prime}=M_{i j} a_{-n}^{i} a_{n}^{j} /\left(2 \alpha^{\prime} n\right)$ and $N_{n}^{\prime \prime}=a_{-n}^{a} a_{n}^{a} /\left(2 \alpha^{\prime} n\right)$ are the number operators for the (properly normalized) oscillation modes and the mass $M$ is given by

$$
M^{2}=-P_{\text {tot }}^{k} P_{\text {tot }}^{k}
$$

The action (1) we started with is obtained from a diffeomorphic-invariant action with background fields by gauge fixing $[17,18]$. Thus we need to impose the constraints

$$
\left(\partial_{\tau} X^{\mu}\right)^{2}+\left(\partial_{\sigma} X^{\mu}\right)^{2}=0, \quad \partial_{\tau} X^{\mu} \partial_{\sigma} X_{\mu}=0
$$

on the physical states. One can extract from this the Virasoro generators as usual, they are

$$
L_{k}=\frac{1}{4 \alpha^{\prime}} \sum_{n \in \mathbb{Z}}\left(M_{i j} a_{k-n}^{i} a_{n}^{j}+a_{k-n}^{a} a_{n}^{a}\right),
$$

and they satisfy

$$
L_{m}^{\dagger}=L_{-m}
$$

as a result of (47). With the usual normal ordering of negative modes preceding the positive modes, the normal-ordered Virasoro generators are defined as

$$
L_{k}=\frac{1}{4 \alpha^{\prime}}: \sum_{n \in \mathbb{Z}}\left(M_{i j} a_{k-n}^{i} a_{n}^{j}+a_{k-n}^{a} a_{n}^{a}\right): .
$$

It is easy to check that they satisfy the standard Virasoro algebra

$$
\left[L_{m}, L_{n}\right]=(m-n) L_{m+n}+\frac{d}{12} m\left(m^{2}-1\right) \delta_{m+n}, \quad d=\text { space-time dimension, }
$$

with a central charge not modified by the presence of $\mathcal{F}$. This is necessary for our quantization to be consistent. As usual, one requires that $L_{k}$ for $k \geqslant 0$ annihilate a physical state upon quantization.

\section{M(atrix) model on a torus}

Consider the case of D2-branes. From (52), for $\mathcal{F}_{01}=\mathcal{F}_{02}=0$, the open string end-point at $\sigma=0$ satisfies

$$
\left[X^{1}(0), X^{2}(0)\right]=2 \pi i \alpha^{\prime} \frac{\mathcal{F}}{1+\mathcal{F}^{2}},
$$


where $\mathcal{F}=\mathcal{F}_{12}$. In the static gauge, the coordinates of the open string end-point is the same as the coordinates of the D-brane world-volume. Therefore, the noncommutativity (68) for the string implies that the D-brane world-volume is a noncommutative space. We will show now that (68) in fact agrees with the matrix model results $[3,4]$.

According to the $T$-duality in string theory, a two-torus of radii $R_{1}, R_{2}$ and background $B$ field flux of $\theta$ is dual to the two-torus of radii $\Sigma_{1}, \Sigma_{2}$ with the background flux of $\tilde{\theta}$, where

$$
\begin{aligned}
& \Sigma_{1}=\frac{l_{s}^{2} R_{2}}{\sqrt{l_{s}^{4} \theta^{2}+R_{1}^{2} R_{2}^{2}}}, \\
& \Sigma_{2}=\frac{l_{s}^{2} R_{1}}{\sqrt{l_{s}^{4} \theta^{2}+R_{1}^{2} R_{2}^{2}}}, \\
& \tilde{\theta}=\frac{-l_{s}^{4} \theta}{l_{s}^{4} \theta^{2}+R_{1}^{2} R_{2}^{2}} .
\end{aligned}
$$

One can derive from this the relation between the $B$ fields in the two dual theories

$$
\tilde{B}=\frac{l_{s}^{2} \tilde{\theta}}{\Sigma_{1} \Sigma_{2}}=-\frac{l_{s}^{2} \theta}{R_{1} R_{2}}=-B .
$$

Consider D0-branes in the background of $\tilde{B}$ on a $T^{2}$ with radii $\Sigma_{i}$ as in (69), (70). By $T$-duality we get $\mathrm{D} 2$-branes on $T^{2}$ with radii $R_{1}, R_{2}$ and a background $B$ field. The mass of the D0-brane is $\left(g_{s}^{\prime} l_{s}\right)^{-1}$, where $g_{s}^{\prime}$ is the string coupling for the dual theory and $l_{s}$ is the string length scale. The mass of the D2-brane according to the DBI action is

$$
M=T_{2} V \sqrt{1+B^{2}},
$$

where $T_{2}=\left((2 \pi)^{2} g_{s} l_{s}^{3}\right)^{-1}$ is the D2-brane tension, and $V=(2 \pi)^{2} R_{1} R_{2}$ is the D2-brane volume. The duality between the D0-brane and the D2-brane implies that

$$
g_{s}^{\prime}=\frac{g_{s} l_{s}^{2}}{R_{1} R_{2} \sqrt{1+B^{2}}} .
$$

According to the M(atrix) model, the D2-brane physics is a gauge theory living on a non-commutative torus $[3,4]$

$$
\left[x_{1}, x_{2}\right]=-2 \pi i \tilde{\theta},
$$

where $x_{i} \in[0,2 \pi)$.

On the other hand, in the static gauge we have $X^{i}=R_{i} x_{i}$ as the coordinates on the D2-brane, so

$$
\left[X_{1}, X_{2}\right]=2 \pi i l_{s}^{2} \frac{B}{1+B^{2}}
$$

on using (69), (70) and (72). This agrees precisely with (68) for $F=0$. 
Although in the matrix model derivation we have assumed the compactification on $T^{2}$, after we get the final result we can take the limit of $\Sigma_{i} \rightarrow 0$ with $B$ fixed. This corresponds to uncompactified D2-brane world-volume. There is an issue about the decompactification. Since the $B$ field is a gauge field, in an infinite space we can make a gauge transformation so that $B=0$. So this seems to say that there is no non-commutativity in the decompactification limit. However, this conclusion is not completely correct because on a D2-brane the term $\mathcal{F}=B-F$ is gauge invariant. So even if $B=0$, there is still non-commutativity if $\mathcal{F} \neq 0$.

It may also appear at first sight that there is a mismatch between the matrix model results and the open string calculation presented here. For matrix model compactified on a torus of radii $\Sigma_{1}, \Sigma_{2}$ with a background flux of $\tilde{\theta}$, the two-dimensional SYM theory lives on a non-commutative torus of radii $l_{s}^{2} / \Sigma_{1}, l_{s}^{2} / \Sigma_{2}[3,14]$, which is different from what is expected from the $T$-duality of string theory, i.e. $R_{1}, R_{2}$. However, this is not a true discrepancy because the torus on which the SYM theory lives is not exactly the torus in the dual string theory. In fact it was shown [19] that if we consider the DBI action for D2-branes on a torus of radii $R_{1}, R_{2}$, its expansion agrees with a SYM action on a torus with radii $l_{s}^{2} / \Sigma_{i}$.

The DBI action for a D2-brane in the background $B$ field is

$$
S=T_{2} \int d t d^{2} \xi \sqrt{-\operatorname{det}(G+\mathcal{F})},
$$

where $G_{i j}=\eta_{i j}+\partial_{i} X^{a} \partial_{j} X_{a}$ is the induced metric, and $\xi_{i} \in\left[0,2 \pi R_{i}\right)$ are the coordinates on the D2-brane world-volume. One can expand the Lagrangian with respect to $\left(1+\mathcal{F}^{2}\right)$ as

$$
\begin{aligned}
\sqrt{-\operatorname{det}(G+\mathcal{F})} & =\sqrt{\left(1+\mathcal{F}^{2}\right)\left(1-\frac{\left(\partial_{i} X^{a}\right)^{2}}{1+\mathcal{F}^{2}}+\ldots\right)} \\
& =\sqrt{1+\mathcal{F}^{2}}-\frac{1}{2 \sqrt{1+\mathcal{F}^{2}}}\left(\partial_{i} X^{a}\right)^{2}+\ldots,
\end{aligned}
$$

thus the DBI action contains a part which is of the same form as the supersymmetric Yang-Mills action

$$
\frac{T_{2}}{\sqrt{1+\mathcal{F}^{2}}} \int d t d^{2} \xi\left(\frac{1}{2}\left(\partial_{i} X^{a}\right)^{2}+\ldots\right) .
$$

If we start with the dual theory of D0-branes on the dual torus $T^{2}$ in the context of $M$ (atrix) compactification, we proceed as follows. The D0-brane SYM action is

$$
S=\frac{1}{g_{s}^{\prime} l_{s}} \int d t\left(\frac{1}{2}\left(\partial_{t} X^{\mu}\right)^{2}+\ldots\right) .
$$

The quotient conditions

$$
U_{i}^{\dagger} X_{j} U_{i}=X_{j}+2 \pi \Sigma_{j} \delta_{i j}, \quad i=1,2
$$


are solved by

$$
X^{i}=-i 2 \pi \frac{\partial}{\partial y_{i}}+A^{i}(y),
$$

where $y \in\left[0,2 \pi l_{s}^{2} / \Sigma_{i}\right)$. To compare this result with the above, set $y_{i}=\sqrt{1+\mathcal{F}^{2}} \xi_{i}$, so the SYM action of D0-brane becomes

$$
S=\frac{1}{g_{s}^{\prime} l_{s}} \int d t \int \frac{d^{2} \xi}{\left(2 \pi l_{s}^{2} / \Sigma_{1}\right)\left(2 \pi l_{s}^{2} / \Sigma_{2}\right)}\left(\frac{1}{2}\left(\partial_{i} X^{a}\right)^{2}+\ldots\right),
$$

where $\partial_{i}=\partial / \partial \xi_{i}$. Using (74), one can see that (79) agrees with (83) when $F=0$.

It is straightforward to generalize the discussion to $\mathrm{D} p$-branes. We get from $(52)$

$$
\left[X^{k}, X^{l}\right]= \pm 2 \pi i \alpha^{\prime}\left(M^{-1} \mathcal{F}\right)^{k l}
$$

with the plus (minus) sign corresponding to the end-point at $\sigma=0(\pi)$. This formula tells us explicitly how the string theory data appear in the non-commutativity of the $\mathrm{D} p$-brane world-volume. As in the D2-brane case, one can show that it agrees with the results of $\mathrm{M}$ (atrix) theory if one uses the $T$-duality radii instead of using the $\mathrm{M}$ (atrix) model radii natively. Let us explain this in more detail. Consider a $\mathrm{D} p$-brane on $T^{p}$ with radii $R_{i}$ in a background $B$-field. The dimensionless metric and flux are

$$
G_{i j}=\delta_{i j} R_{i}^{2} / 2 \alpha^{\prime}, \quad \theta_{i j}=B_{i j} R_{i} R_{j} / 2 \alpha^{\prime},
$$

where $i, j=1, \ldots, p$ and there is no sum of indices. This is the theory of interest. The $T$-dual theory is D0-branes on a $T^{p}$ with radii $\Sigma_{i}$ in a background of $\tilde{B}$. The dimensionless metric $\tilde{G}$ and flux $\tilde{\theta}$ are (set $2 \alpha^{\prime}=1$ for convenience)

$$
\tilde{G}+\tilde{\theta}=(G+\theta)^{-1}
$$

(see Ref. [20] for a review of $T$-duality). Denote

$$
(G+\theta)_{i j}=R_{i} R_{j}\left(\delta_{i j}+B_{i j}\right)=R_{i} R_{j} g_{i j}
$$

and so

$$
\tilde{G}_{i j}+\tilde{\theta}_{i j}=\frac{1}{R_{i} R_{j}} g_{i j}^{-1}
$$

It is

$$
g^{-1}=(1+B)^{-1}=\left(1+B^{2}+B^{4}+\ldots\right)-\left(B+B^{3}+\ldots\right),
$$

where the first sum is symmetric and the second sum is antisymmetric. Define

$$
m_{i}^{j}=\delta_{i}^{j}-B_{i}{ }^{k} B_{k}{ }^{j}
$$

This is $M$ for the case of $A=0$. Then

$$
g^{-1}=m^{-1}-B m^{-1} \text {. }
$$


Hence

$$
\tilde{G}_{i j}=\frac{1}{R_{i} R_{j}} m_{i j}^{-1}, \quad \tilde{\theta}_{i j}=-\frac{1}{R_{i} R_{j}}\left(B m^{-1}\right)_{i j} .
$$

Generalizing the arguments in $[3,4]$, it is easy to see that $\mathbf{M}$ (atrix) model predicts in general

$$
\left[x^{i}, x^{j}\right]=-2 \pi i \tilde{\theta}^{i j},
$$

where $x^{i} \in(0,2 \pi)$ is the angular coordinates of the $\mathrm{D} p$-brane. Substituting (92) into (93), we get

$$
\left[X^{i}, X^{j}\right]=2 \pi i \alpha^{\prime}\left(m^{-1} B\right)^{i j} \quad \text { with } X^{i}=R_{i} x^{i},
$$

which is precisely our result for the case of $A=0$. From the $\mathrm{D} p$-brane point of view, it is natural to expect that it is $\mathcal{F}$ (instead of $B$ ) that controls the non-commutativity of the world-volume field theory. We will now explain the reason for the signs in (84) from the point of view of D-brane world-volume theory.

\section{D-brane field theory}

Consider the D2-brane case for simplicity, the other end-point of the open string at $\sigma=\pi$ satisfies

$$
\left[X^{1}(\pi), X^{2}(\pi)\right]=-2 \pi i \alpha^{\prime} \frac{\mathcal{F}}{1+\mathcal{F}^{2}} .
$$

Note that there is only a difference in sign on the right-hand side when compared with (68). We will now show that this sign difference is important for the gauge field theory on the D-brane world-volume to exist.

Let us summarize (68), (95) as

$$
\left[x_{1}^{ \pm}, x_{2}^{ \pm}\right]=i h^{ \pm},
$$

where $x_{i}^{-}=X_{i}(0)$ and $x_{i}^{+}=X_{i}(\pi)$ denote the two end-points of the open string at $\sigma=0$ and $\sigma=\pi$, and $h^{ \pm}= \pm 2 \pi \alpha^{\prime} \mathcal{F} /\left(1+\mathcal{F}^{2}\right)$. The coordinates on the D2-brane can be identified with the coordinates of the end-points of the open string. It may seem at first that for both end-points of a string to end on the same D2-brane we need $h^{+}=h^{-}$ in order to have a unique commutation relation for the D2 world-volume coordinates, but below we argue that this condition should be instead

$$
h^{+}=-h^{-} \text {, }
$$

otherwise we do not know how to describe the D2-brane gauge field theory on a single non-commutative space. 
The end-points of open strings are described by a complex field on the D2-brane because there are both positive and negative charges. For instance the fermionic ground state on the open string is described in the D2-brane field theory by a fermionic field,

$$
\psi(x)=\sum_{n}\left(a_{n}^{\dagger}(x) e^{i w_{n} t}+b_{n}(x) e^{-i w_{n} t}\right)
$$

and similarly for bosonic modes. The interaction between $\psi$ and $A_{i}$ on D2-brane is given by a term like $L_{\mathrm{int}}=\bar{\psi} \gamma^{i} A_{i} \psi$ in the Lagrangian. In this term the field $A(x)$ acts on $a^{\dagger}(x)$ from the left and it acts on $b^{\dagger}(x)$ from the right. To bring the action of the $A_{i}$ field on the two charges to the same form, we define $A^{\prime}$ from $A$ by

$$
A^{\prime}\left(x^{\prime}\right)=\sum A_{m n} e^{i n x_{2}^{\prime}} e^{i m x_{1}^{\prime}},
$$

if $A$ is given by $A(x)=\sum A_{m n} e^{i m x_{1}} e^{i n x_{2}}$. The coordinates $x_{i}^{\prime}$ are operators just like $x_{i}$. They are defined by multiplication from the right,

$$
x_{i}^{\prime} \psi(x)=\psi(x) x_{i}
$$

so $x^{\prime}$ satisfies the opposite algebra of $x$. Now the interaction term can be written in terms of $A$ acting on $a^{\dagger}$ and $A^{\prime}$ acting on $b^{\dagger}$ both from the left. In this convention we see that the gauge field seen by the positively charged end-points, which correspond to $a^{\dagger}$, is $A(x)$, and the gauge field seen by the negatively charged end-points, which correspond to $b^{\dagger}$, is $A^{\prime}\left(x^{\prime}\right)$. This indicates that we have $x \sim x^{+}$and $x^{\prime} \sim x^{-}$. Since $x^{\prime}$ is the opposite algebra of $x$, we need (97).

It follows from (100) that $x^{\prime}$ commutes with $x$. This is consistent with the fact that the two end-points of an open string commute

$$
\left[X^{i}(0), X^{j}(\pi)\right]=0
$$

which follows directly from (52).

The physical reason for (97) is just that the two end-points have opposite charges under $\mathcal{F}$ and $h^{ \pm}$is proportional to $\mathcal{F}$. So the symmetry of charge conjugation is preserved only if (97) holds.

In the absence of background fields $B$ and $F$, the low energy physics of $N$ coincident $\mathrm{D} p$-branes is given by the $U(N) \mathrm{SYM}$ theory dimensionally reduced to $p+1$ dimensions. In Ref. [7], it was proposed that the gauge field theory for D-branes is also given by SYM theory on a quantum plane when the background $B$ field exists, just like the case in matrix theory. Since the matrix model is related to the D-brane physics by the Seiberg limit [21], it is not at all obvious that this statement is correct. In fact, there is a serious mismatch for this interpretation [7], that is, the $S L(2, \mathbb{Z})$ transformation for the $B$ field in such a theory is correct only in the matrix model limit. Nevertheless, our analysis of the open string quantization shows that the proposal of [7] must be correct in the sense that the field theory of the D-brane must be a field theory on a non-commutative space, although we might be still missing something to fill the noted gap. 


\section{Remarks}

\subsection{Fermionic modes}

In the above we have only focused on the bosonic modes on the open string. Now we consider the fermionic modes. In the RNS string action, the fermionic part is

$$
S_{F}=-\frac{i}{4 \pi \alpha^{\prime}} \int d^{2} \sigma\left(\bar{\psi}^{\mu} \rho^{\alpha} \partial_{\alpha} \psi_{\mu}\right)
$$

It is not modified by a constant $B$ field. The supersymmetry on the world sheet is

$$
\delta X^{\mu}=\bar{\epsilon} \psi^{\mu}, \quad \delta \psi^{\mu}=-i \rho^{\alpha} \partial_{\alpha} X^{\mu} \epsilon .
$$

The equations of motion,

$$
\rho^{\alpha} \partial_{\alpha} \psi^{\mu}=0
$$

and the boundary conditions,

$$
\psi_{+}^{\mu}= \pm \psi_{-}^{\mu}
$$

at $\sigma=0, \pi$ are the same as before. Therefore the fermionic fields on the open string are exactly the same as when $\mathcal{F}$ is zero. The two choices of sign in the boundary conditions lead to the Ramond sector and the Neveu-Schwarz sector which give the ground states of a spinor and a vector, respectively. They correspond to the fermionic field and the gauge field in the SYM theory in $9+1$ dimensions. For the $\mathrm{D} p$-brane field theory the gauge field is dimensionally reduced to the gauge field $A_{i}$ and the Higgs $X_{a}$. It is therefore quite trivial to include the fermionic modes in all our discussion above.

\subsection{Generalizations}

It is clear from our derivation that the non-commutativity can exist for generic geometry or topology of the $\mathrm{D} p$ brane. For example, a D2-brane can be a non-commutative $S^{2}$ or $S^{1,1}$. For these cases the commutation relations for the oscillation modes may be difficult to derive, but for the lowest modes we may use symmetry requirements to fix their relations, assuming that the $\mathcal{F}$ field also respects part of the isometry group. For instance, for a spherical D2-brane, the end-point of an open string in Cartesian coordinates satisfies

$$
\left[X_{i}, X_{j}\right]=i h \epsilon_{i j k} X_{k},
$$

where $h=h(\mathcal{F})$ is a constant satisfying $h(0)=0$. The point is that the $\mathcal{F}$ field determines the symplectic structure and if it respects the isometry of the space it may be fixed by the symmetry up to an overall factor.

In this paper, we considered string ending on a $\mathrm{D} p$-brane and showed that the endpoints become non-commutative in background fields. Using $S$-duality, one can turn 
this into a configuration of a D1-brane ending on a $\mathrm{D} p$-brane with a background $\mathrm{R}-\mathrm{R}$ $B$-field. The end-points of the D1-brane and hence the world-volume of the Dp-brane will again have non-commutative coordinates. Combining $S$-duality and $T$-duality, one can arrive at more general configurations of branes ending on branes. For example, a solitonic brane ending on a D-brane; or a $\mathrm{D} p$-brane ending on a $\mathrm{D} q$-brane. In these cases, the end-points of the "smaller" brane and hence the "host brane" world-volume will again have non-commutative coordinates, although showing it directly may be difficult due to complications in quantization of higher dimensional branes and solving the corresponding boundary conditions which are no longer linear for higher dimensional extended objects. Nevertheless, the lowest energy modes can still be dealt with. Let us discuss some features in a similar classical spirit as in [23].

For instance for a membrane ending on a M5-brane, the boundary conditions analogous to (4) are

$$
\partial_{2} X^{i}-\mathcal{F}^{i}{ }_{j k} \partial_{0} X^{j} \partial_{1} X^{k}=0,
$$

where $a, b=0,1,2$ are the membrane world-volume index. This boundary condition can be derived from the bosonic action

$$
S=\int d^{3} \sigma\left(\frac{1}{2}\left(\partial_{a} X^{i}\right)^{2}-\frac{1}{6} \epsilon^{a b c} \mathcal{F}_{i j k} \partial_{a} X^{i} \partial_{b} X^{j} \partial_{c} X^{k}\right) .
$$

At the boundary of the membrane, $X^{k}$ can be identified with the M5-brane world-volume and $\mathcal{F}$ is the modified field strength on the M5-brane. The non-linearity of the boundary conditions makes it difficult to obtain a generic solution of $X^{i}$. It is however possible to examine the lowest energy modes

$$
X^{i}=x^{i}+p_{a}^{i} \sigma_{a}+\mathcal{F}^{i}{ }_{j k} p_{0}^{j} p_{1}^{k} \sigma_{2}+\ldots
$$

Choosing the range of $\sigma_{2}$ to be $[-\pi / 2, \pi / 2]$, one finds the $\tilde{\Omega}$ for these modes

$$
\tilde{\Omega}=\left(\mathbf{d} p_{0}^{i}-\mathcal{F}^{i}{ }_{j k} \mathcal{F}_{l m}^{k} \mathbf{d}\left(p_{0}^{l} p_{1}^{j} p_{1}^{m}\right)\right) \mathbf{d} x_{i} .
$$

One can check that when $X^{1}$ is compactified on a circle of radius $R$, this reduces to the case for an open string with $\mathcal{F}_{i j}=\mathcal{F}_{i j k} p_{1}^{k}$.

\subsection{Uncertainty relation}

In the second quantization of string theory, one has to integrate over all possible configurations. So even if we consider a classical background of vanishing VEV for $\mathcal{F}$, in general

$$
\int[D \mathcal{F}][D \Psi] e^{-S(\mathcal{F}, \Psi)}\left(\Delta X_{1}\right)^{2}\left(\Delta X_{2}\right)^{2}>0,
$$

will not be zero and non-commutativity on the world-volume of extended objects is a generic feature of string theory. In (111), $S$ is the action of string field theory. $\Psi$ is the wave function for D-brane and $\left(\Delta X_{i}\right)^{2}$ and $\left\langle X_{i}(\sigma, t)\right\rangle$ are defined by 


$$
\begin{aligned}
\left(\Delta X_{i}\right)^{2} & =\int[D X(\sigma, t)] \Psi(X)^{\dagger}\left(X_{i}(\sigma, t)-\left\langle X_{i}(\sigma, t)\right\rangle\right)^{2} \Psi(X), \\
\left\langle X_{i}(\sigma, t)\right\rangle & =\int[D X(\sigma, t)] \Psi^{\dagger} X_{i}(\sigma, t) \Psi .
\end{aligned}
$$

It would be interesting [25] if some connection of this with the work of [24] can be established.

\subsection{Conclusion}

In this paper, we show that non-commutativity of the D-brane world-volume is a direct consequence of quantizing open string theory in non-zero backgrounds of $B-F$. The quantization we proposed is a consistent quantization which covers and generalizes the usual situations, and the unitary flow equation is reproduced. Moreover, we show that it agrees with the non-commutativity obtained in $\mathbf{M}$ (atrix) model compactification provided we use the $T$-duality radii. Our results however generalize the M(atrix) model results in several ways.

In the $\mathbf{M}$ (atrix) model, non-commutativity was argued to exist on compactification. Here, we showed that not only we do not need compactification for non-commutativity, in general we have non-commutativity whenever $B-F$ is non-zero on a D-brane. Also we give the explicit result ( 84 ) of how non-commutativity should look like for higher dimensional $\mathrm{D} p$-branes. The data involved is the gauge-invariant $\mathcal{F}$ instead of the $B$ as suggested in $\mathrm{M}$ (atrix) model compactification. It would be very interesting to see this directly from the M(atrix) model compactification or from another point of view [25].

In a string field theory one has a Hilbert space that contains all the states of string theory, including the closed strings, open strings and D-branes. A closed string can break itself and end on a D-brane. It can also break itself and interact with another closed string or open string. One may perhaps anticipate that non-commutativity is not confined to the world-volume of D-branes, but is generic to all extended objects in a consistent formulation of string theory.

\section{Acknowledgements}

C.S.C. thanks A. Bilal, M. Cederwall, C. Hull and R. Russo for helpful discussions. P.M.H. thanks M.M. Sheikh-Jabbari for valuable discussions and the Asia Pacific Center for Theoretical Physics for hospitality where part of this work was done. The work of C.S.C. is supported by the Swiss National Science Foundation. The work of P.M.H. is supported in part by the National Science Council, Taiwan, R.O.C.

\section{References}

[1] T. Banks, W. Fischler, S.H. Shenker and L. Susskind, M-theory as a matrix model, a conjecture, Phys. Rev. D 55 (1997) 5112, hep-th/9610043. 
|2| W. Taylor, D-brane field theory on compact spaces, Phys. Lett. B 394 (1997) 283, hep-th/961 1042.

[3] A. Connes, M.R. Douglas and A. Schwarz, Non-commutative geometry and matrix theory, compactification on tori, J. High Energy Phys. 02 (1998) 003, hep-th/9711162.

[4] M.R. Douglas and C. Hull, D-branes and non-commutative torus, J. High Energy Phys. 02 (1998) 008 , hep-th/9711165.

[5] Y.-K.E. Cheung and M. Krogh, Non-commutative geometry from 0-branes in a background $B$-field, Nucl. Phys. B 528 (1998) 185, hep-th/9803031.

[6] T. Kawano and K. Okuyama, Matrix theory on non-commutative torus, Phys. Lett. B 433 (1998) 29 , hep-th/9803044.

[7] C. Hofman and E. Verlinde, $U$-duality of Born-Infeld on the non-commutative two-torus, hepth/9810116.

[8] F. Ardalan, H. Arfaei and M.M. Sheikh-Jabbari, Mixed branes and M(atrix) theory on non-commutative torus, hep-th/9803067; Non-commutative Geometry from Strings and Branes, hep-th/9810072.

[9] J. Dai, R.G. Leigh and J. Polchinski, New connections between string theories, Mod. Phys. Lett. A 4 (1989) 2073.

[10] R. Leigh, Dirac-Born-Infeld action from Dirichlet sigma models, Mod. Phys. Lett. A 4 (1989) 2767.

[11] P. Di Vecchia, M. Frau, I. Pesando, S. Sciuto, A. Lerda and R. Russo, Classical $p$-branes from boundary state, Nucl. Phys. B 507 ( I997) 259, hep-th/9707068.

[12] H. Arfaei and M.M. Sheikh-Jabbari, Mixed boundary conditions and brane-string bound states, Nucl. Phys. B 526 (1998) 278, hep-th/9709054.

I 13] P.-M. Ho and Y.-S. Wu, Non-commutative gauge theories in matrix theory, hep-th/9801147.

[14] P.-M. Ho, Twisted bundle on quantum torus and BPS states in matrix theory, hep-th/9803166.

[15] M.M. Sheikh-Jabbari, More on mixed boundary conditions and D-brane bounded states, Phys. Lett. B 425 (1998) 48, hep-th/9712199.

[16] E. Witten, Non-abelian bosonization in two dimensions, Comm. Math. Phys. 92 (1984) 455.

[17] A. Das, J. Maharana and S. Roy, BRST quantization of the superstring in background fields, Phys. Rev. D 40 (1989) 4037.

[18] S. Alam, Becchi-Rouet-Stora-Tyutin quantization of closed type-ll superstring in curved background with torsion, Phys. Rev. D 40 (1989) 4047.

119| M. Li, Comments on supersymmetric Yang-Mills theory on a non-commutative torus, hep-th/9802052.

[20] A. Giveon, M. Porrati and E. Rabinovici, Target space duality in string theory, Phys. Rep. 244 (1994) 77 , hep-th/9401139.

[21] N. Seiberg, Why is the matrix model correct?, Phys. Rev. Lett. 79 (1997) 3577, hep-th/9710009.

122] J. Polchinski, S. Chaudhuri and C.V. Johnson, Notes on D-branes, hep-th/9602052.

123] M.J. Duff, P.S. Howe, T. Inami and K.S. Stelle, Superstrings in $D=10$ from supermembrane in $D=11$, Phys. Lett. B 191 (1987) 70.

[24] M. Li and Y. Yoneya, D-particle dynamics and the space-time uncertainty relation, Phys. Rev. Lett. 78 (1997) 1219, hep-th/9611072;

A. Jevicki and T. Yoneya, Space-time uncertainty principle and conformal symmetry in D-particle dynamics, Nucl. Phys. B 535 (1998) 335, hep-th/9805069.

125] C.-S. Chu and P.-M. Ho, work in progress. 